\title{
Achados oculares e fundoscópicos em pacientes com lupus eritematoso sistêmico
}

\author{
Ocular and fundoscopic findings in patients with systemic lupus erythematosus
}

\author{
Silvane Bigolin ${ }^{(1)}$ \\ Emerson Oyamaguchi ${ }^{(2)}$ \\ Cláudia del Claro ${ }^{(1)}$ \\ Agostinho Bryk Junior ${ }^{(3)}$ \\ Maria Cláudia Gomes Komatsu ${ }^{(3)}$ \\ Eduardo Belotto ${ }^{(4)}$ \\ Ezequiel Postella ${ }^{(5)}$
}

\begin{tabular}{|c|}
\hline RESUMO \\
\hline $\begin{array}{l}\text { Objetivos: Descrever as alterações encontradas no exame ocular } \\
\text { de pacientes com lupus eritematoso sistêmico (LES), especialmente } \\
\text { aquelas relacionadas à fundoscopia. } \\
\text { Método: Estudo descritivo de } 41 \text { pacientes lúpicos, selecionados } \\
\text { aleatoriamente entre maio e julho de } 1999 \text { no ambulatório de } \\
\text { colagenoses do Serviço de Reumatologia do Hospital de Clínicas da } \\
\text { Universidade Federal do Paraná. Foi aplicado um protocolo de } \\
\text { avaliação clínica e ocular. } \\
\text { Resultados: Foram incluídos no estudo } 41 \text { pacientes, sendo } 40 \\
\text { (97,56\%) do sexo feminino e } 01 \text { (2,44\%) do sexo masculino. A média } \\
\text { de idade foi de } 32,22 \text { anos, com mínima de } 16 \text { e máxima de } 54 \text { anos. } \\
\text { Trinta e seis pacientes (87,80\%) eram de cor branca, dois (4,89\%) de } \\
\text { cor negra e três (7,32\%) pardos. O tempo de diagnóstico de LES } \\
\text { variou de dois meses a } 18 \text { anos. Dos } 41 \text { pacientes, } 19 \text { (46,34\%) } \\
\text { apresentaram alteração fundoscópica relacionada ao LES. As prin- } \\
\text { cipais lesões encontradas foram manchas algodonosas e estreitamento } \\
\text { arteriolar (68,42\%), seguidas de aumento da escavação (10,52\%) e } \\
\text { palidez do disco óptico (10,52\%), lesão perivascular (5,26\%) e alte- } \\
\text { ração no epitélio pigmentar da retina (5,26\%). } \\
\text { Conclusão: Observou-se alta prevalência de alterações } \\
\text { fundoscópicas relacionadas ao LES, demonstrando a importância de } \\
\text { exames fundoscópicos regulares mesmo em pacientes lúpicos } \\
\text { assintomáticos ou sem doenças associadas. }\end{array}$ \\
\hline
\end{tabular}

Palavras chave: Lupus eritematoso sistêmico; Fundoscopia.
Trabalho desenvolvido no Serviço de Oftalmologia do Hospital de Clínicas da Universidade Federal do Paraná.

Apresentado como tema livre no XXIV Simpósio da Associação Paranaense de Oftalmologia em 03/12/99 em Curitiba - PR

(1) Acadêmicas do curso de medicina da Universidade Federal do Paraná.

(2) Médico residente do serviço de Oftalmologia do Hospital de Clínicas da Universidade Federal do Paraná. Acadêmicos do curso de medicina da Pontifícia Universidade Católica do Paraná.

4) Médico residente do serviço de Reumatologia do Hospital de Clínicas da Universidade Federal do Paraná.

5) Médico oftalmologista chefe do ambulatório de retina e vítreo do serviço de Oftalmologia do Hospital de Clínicas da Universidade Federal do Paraná. Os autores declaram não possuir interesse financeiro no desenvolvimento ou marketing de qualquer instrumento referido.

Endereço para correspondência: Silvane Bigolin - Av. Silva Jardim, 1514 ap. 1601 Rebouças, Curitiba- PR. CEP: 80250-200. Fone: 41-322-3171. Email: silvane.bigolin@uol.com.br

\section{INTRODUÇÃOO}

O lupus eritematoso foi descrito em primeira instância em 1833 por Brett. Durante décadas acreditou-se tratar de uma doença dermatológica crônica. Em 1872, Kaposi descreveu o lupus eritematoso sistêmico, sendo que desde esta data várias alterações em diferentes órgãos foram descritas ${ }^{1}$.

O lupus eritematoso sistêmico é uma doença de origem desconhecida que envolve diferentes sistemas do organismo, caracterizada pela formação de auto-anticorpos, deposição de imunocomplexos e oclusão de pequenos vasos em órgãos variados ${ }^{1,2}$. A deposição vascular de imunocomplexos leva à ativação da cascata do complemento, gerando vasculite ${ }^{2}$. As manifestações clínicas mais comuns são poliartrite, erupção cutânea, febre e desordens neuropsiquiátricas. Anormalidades imunológicas são bastante freqüentes, particularmente a presença de anticorpos anti-nucleares ${ }^{2}$.

As manifestações oftálmicas do lupus eritematoso sistêmico variam 
desde acometimento das pálpebras pela doença mucocutânea até doença vascular retiniana e envolvimento neuro-oftálmico ${ }^{3-5}$.

O LES é uma doença caracterizada pela deposição de imunocomplexos em órgãos alvo. O sinal histológico patognomônico da retinopatia lúpica é a inflamação de pequenos vasos retinianos que são obliterados por material amorfo formando áreas de necrose fibrinóide e hialinização. Células inflamatórias não são vistas freqüentemente, sugerindo que as lesões são causadas por depósitos locais de complexos imunes ${ }^{7}$.

As manifestações fundoscópicas do LES geralmente consistem de manchas algodonosas com ou sem hemorragias intraretinianas, papiledema, hiperemia e edema retinianos, os quais podem ocorrer mesmo na ausência de hipertensão intracraniana. Estudos histológicos revelam que a coróide pode apresentar-se infiltrada por leucócitos e que tal infiltração pode ser tão severa a ponto de causar destruição das camadas retinianas profundas. Grandes descolamentos retinianos serosos, atrofia óptica e perda visual súbita têm sido relatados. Oclusão das grandes arteríolas por espasmo e trombose também são descritos e ocorrem associados à vasculite ${ }^{7}$.

Este estudo tem como objetivo descrever as alterações encontradas no exame ocular de pacientes com LES, especialmente aquelas relacionadas à fundoscopia.

\section{PACIENTES E MÉTODOS}

Constituiu-se de um estudo descritivo no qual foram avaliados 41 pacientes com diagnóstico de lupus eritematoso sistêmico em acompanhamento no ambulatório de colagenoses do serviço de Reumatologia do Hospital de Clínicas da Universidade Federal do Paraná, selecionados aleatoriamente no período de maio a julho de 1999. Os pacientes preenchiam no mínimo quatro critérios de classificação do lupus eritematoso sistêmico do American College of Rheumatology de 1982 . Foi aplicado um protocolo que continha história clínica detalhada incluindo tempo de diagnóstico, envolvimento sistêmico, doenças associadas, queixas oculares, uso de medicações anti-lúpicas atuais ou anteriores e perfil de autoanticorpos (fator antinuclear, anti Sm, anti RNP, anti Ro, anti $\mathrm{La}$, anticardiolipina e anticoagulante lúpico). Foram considerados com hipertensão arterial sistêmica os pacientes em cujo prontuário médico constava a informação da pressão arterial com níveis sistólicos maiores ou iguais a $140 \mathrm{mmHg}$ ou níveis diastólicos maiores ou iguais a $90 \mathrm{mmHg}$, no mínimo em duas consultas.

Os pacientes foram submetidos ao exame ocular no Serviço de Oftalmologia da mesma instituição sempre pelo mesmo examinador. Tal exame incluiu determinação da acuidade visual pela tabela de Snelen, tonometria através do tonômetro de não contato Topcon AIT $20 \mathrm{CT}$, biomicroscopia através da lâmpada de fenda Topcon SL 7E e tela de Amsler. Seguiu-se a instilação de uma gota de Mydriacil 1\% ${ }^{\circledR}$ e uma gota de fenilefrina $10 \%$ em ambos os olhos, com intervalo de cinco minutos entre a aplicação dos dois colírios. Após um período mínimo de 20 minutos foi realizada a fundoscopia através do oftalmoscópio indireto Topcon PS 12, com lentes de 20 e 90 dioptrias.

\section{RESULTADOS}

Foram incluídos no estudo 41 pacientes, sendo 40 $(97,56 \%)$ do sexo feminino e um $(2,44 \%)$ do sexo masculino. A média de idade dos pacientes foi de 32,22 anos, com mínima de 16 e máxima de 54 anos. Trinta e seis pacientes $(87,80 \%)$ eram de cor branca, dois $(4,89 \%)$ de cor negra e três $(7,32 \%)$ pardos.

O tempo de diagnóstico do lupus eritematoso sistêmico variou de dois meses a 18 anos, com uma média de 6,17 anos. A distribuição do envolvimento sistêmico encontrado nos pacientes pode ser observada na tabela 1 . Dez pacientes (24,39\%) apresentavam diagnóstico de hipertensão arterial sistêmica (HAS), sendo que destes, sete (70\%) faziam uso de um ou mais medicamentos anti-hipertensivos. A distribuição de pacientes hipertensos segundo o envolvimento sistêmico pode ser observada na tabela 1 .

No estudo do perfil de anticorpos, quatro pacientes $(9,97 \%)$ tiveram todas as pesquisas para anticorpos relacionados ao lupus negativas. Foram encontrados 33 pacientes $(80,49 \%)$ com FAN (fator anti-nuclear) positivo, cinco $(12,20 \%)$ com perfil ENA(anti Ro, anti La, anti Sm, anti RNP) positivo, dois $(4,89 \%)$ com anticardiolipina positivo e dois (4,89\%) com anti-lúpico positivo.

Trinta e oito pacientes apresentavam uso atual de medicação anti-lúpica sendo que destes, 12 (31,58\%) faziam uso de corticóide, cinco $(13,16 \%)$ faziam uso de cloroquina, 16 $(42,11 \%)$ usavam corticóide associado à cloroquina e cinco $(13,16 \%)$ usavam a associação de corticóide, cloroquina e ciclofosfamida.

Onze pacientes apresentavam queixas oculares. Destes, prurido foi a queixa mais freqüente, relatada por seis pacientes $(54,55 \%)$. Quatro pacientes $(36,36 \%)$ queixaram-se de ardência ocular, três $(27,27 \%)$ de hiperemia ocular, dois $(18,18 \%)$ de lacrimejamento e um $(9,09 \%)$ queixou-se de mosca volante.

Tabela 1. Correlação entre envolvimento sistêmico do lupus eritematoso sistêmico com hipertensão arterial sistêmica (HAS).

\begin{tabular}{lcc|} 
Sistema comprometido & $\begin{array}{c}\text { Número de pacientes } \\
\mathbf{N = 4 1}\end{array}$ & $\begin{array}{c}\text { HAS } \\
\mathbf{N = 1 0}\end{array}$ \\
Articular & $40(97,56 \%)$ & 10 \\
Cutâneo & $37(90,24 \%)$ & 10 \\
Renal & $19(46,34 \%)$ & 09 \\
Hematológico & $11(26,83 \%)$ & 03 \\
Vascular & $11(26,83 \%)$ & 03 \\
Sistema nervoso central & $09(21,95 \%)$ & 04 \\
Serosa & $06(14,63 \%)$ & 03 \\
Cardíaco & $02(04,89 \%)$ & 02 \\
Alterações fundoscópicas & $23(56,10 \%)$ & 08 \\
Outros sistemas & $01(02,44 \%)$ & 00 \\
\hline
\end{tabular}


Em todos os pacientes o exame de inspeção ocular, motilidade ocular extrínseca e intrínseca não mostraram alterações.

A distribuição da acuidade visual encontrada nos 41 pacientes ( 82 olhos) é mostrada na tabela 2 .

A média de pressão intra-ocular foi de $16,82 \mathrm{mmHg}$, com mínima de 12 e máxima de $25 \mathrm{mmHg}$. O valor considerado normal no estudo foi de até $22 \mathrm{mmHg}$.

No exame de biomicroscopia, foram encontradas alterações em 19 pacientes (46,34\%) sendo que destes, 12 $(63,16 \%)$ apresentavam blefarite, três $(15,79 \%)$ pterígeo, dois $(10,53 \%)$ calásio, um $(5,26 \%)$ apresentava catarata subcapsular posterior e um $(5,26 \%)$ ceratite puntata.

Foram encontradas alterações na fundoscopia de 19 pacientes (46,34\%). As lesões encontradas e sua distribuição são mostrados na tabela 3. Dos pacientes com anticorpos anticardiolipina positivos, nenhum apresentou alterações fundoscópicas.

Duas pacientes apresentaram aumento da escavação da papila, ambas com história prévia de uso de corticóide sistêmico em doses elevadas por mais de três anos (sem uso atual) e sem história pregressa ou familiar de galucoma. A primeira, cuja idade no momento do exame era de 37 anos, apresentou pressão intra-ocular de $23 \mathrm{mmHg}$ e acuidade visual de 20/60 em ambos os olhos, sem melhora com a refração. A segunda paciente, 42 anos, apresentou PIO de 24 $\mathrm{mmHg}$ no olho direito, $22 \mathrm{mmHg}$ no olho esquerdo e acuidade

\begin{tabular}{|lc|}
\hline $\begin{array}{l}\text { Tabela 2. Distribuição dos pacientes segundo a acuidade visual } \\
\text { Acuidade visual }\end{array}$ & $\begin{array}{c}\text { Número de olhos } \\
\mathbf{N}=\mathbf{8 2}\end{array}$ \\
$20 / 20$ & $34(41,46 \%)$ \\
$20 / 25$ & $18(21,95 \%)$ \\
$20 / 30$ & $08(09,77 \%)$ \\
$20 / 40$ & $08(09,77 \%)$ \\
$20 / 60$ & $06(07,32 \%)$ \\
$20 / 80$ & $03(03,66 \%)$ \\
$20 / 100$ & $02(02,44 \%)$ \\
$20 / 200$ & $02(02,44 \%)$ \\
Contagem de dedos a 4 metros & $01(01,22 \%)$ \\
\hline
\end{tabular}

Tabela 3. Alterações fundoscópicas encontradas em pacientes Lúpicos

\begin{tabular}{|lc|}
$\begin{array}{l}\text { Alteração de fundoscopia } \\
\text { relacionadas ao LES }\end{array}$ & $\begin{array}{c}\text { Número de pacientes } \\
\mathbf{N = 1 9}\end{array}$ \\
$\begin{array}{l}\text { Manchas algodonosas e } \\
\text { estreitamento arteriolar }\end{array}$ & $13(68,42 \%)$ \\
$\begin{array}{l}\text { Atrofia do epitélio pigmentar } \\
\text { retiniano peripapilar }\end{array}$ & $1(5,26 \%)$ \\
$\begin{array}{l}\text { Aumento da escavação do } \\
\text { disco óptico }\end{array}$ & $2(10,52 \%)$ \\
Palidez do disco óptico & $2(10,52 \%)$ \\
Lesão perivascular & $1(5,26 \%)$ \\
\hline
\end{tabular}

visual de 20/80 para o olho direito e 20/60 para o olho esquerdo, sem melhora com a refração.

Dois pacientes apresentavam palidez de papila em ambos os olhos, sendo a acuidade visual de 20/100 em ambos os olhos para o primeiro e de $20 / 80$ no olho direito e de $20 / 200$ no olho esquerdo para o segundo, ambos os pacientes sem melhora com a refração.

\section{DISCUSSÃO}

De acordo com relatos da literatura, a prevalência de alterações retinianas decorrentes do lupus varia de três a $50 \%$ dos pacientes ${ }^{8,9}$. O presente estudo mostrou uma prevalência de 46,34\% (19 pacientes), estando de acordo com a literatura. Embora quatro pacientes apresentassem cicatriz coriorretiniana, esta foi achado de exame e não está correlacionada ao lupus ${ }^{6}$.

As alterações fundoscópicas mais freqüentes neste estudo foram manchas algodonosas e estreitamento arteriolar em 13 pacientes $(68,42 \%)$, as quais são sugestivas de HAS ${ }^{6}$. No entanto, verificou-se que deste grupo de pacientes, seis $(46,15 \%)$ não apresentavam HAS o que leva à atribuição destas lesões primariamente ao LES ${ }^{10}$.

Dois pacientes $(10,52 \%)$ apresentavam aumento da escavação da papila. Sabe-se que o glaucoma é uma complicação do tratamento com corticóides, especialmente os de uso tópico. Entretanto, é descrito que alguns pacientes possam apresentar alterações glaucomatosas após o uso de corticóides sistêmicos ${ }^{3}$, o que sugere que o corticóide sistêmico usado pelos pacientes do estudo possa estar envolvido na gênese do aumento da escavação. Dois pacientes apresentaram palidez de papila. Relatos mostram que cerca de 1 a $2 \%$ dos pacientes lúpicos apresentam neurite ou isquemia óptica e esta manifestase como perda visual progressiva e palidez de papila ${ }^{11}$. Em nenhum paciente foi encontrado edema de papila, o qual tem sido descrito como resultado de uma vasculite dos vasos do nervo óptico ou por envolvimento lúpico no sistema nervoso central, levando a um aumento da pressão intra-craniana e pseudo-tumor orbitário ${ }^{3}$. Foi observada atrofia do epitélio pigmentar retiniano em um paciente, mas não foi possível determinar se foi achado ocasional de exame ou decorrente da infiltração leucocitária na coróide, ocasionando alterações nas camadas mais profundas da retina e do epitélio pigmentar da retina ${ }^{7}$. Em um paciente notou-se lesão perivascular, a qual pode ser decorrente do LES e pode futuramente evoluir para a formação de mancha algodonosa.

Pacientes com LES e com a presença de altos títulos de anticorpos anticardiolipina tem um risco maior de desenvolver doença vascular ocular oclusiva ${ }^{9}$. Neste estudo somente dois pacientes tiveram tais anticorpos positivos, entretanto eles não apresentavam alterações fundoscópicas.

Verificou-se neste estudo alta prevalência de alterações fundoscópicas relacionadas ao LES, o que demonstra a necessidade de avaliações fundoscópicas regulares mesmo em pacientes assintomáticos ou sem doenças associadas, visando 
diagnóstico e intervenção precoces e diminuição da morbidade ocular relacionada a esta doença.

\section{SUMMARY}

Purpose: To describe the changes in ocular examination of patients with systemic lupus erythematosus (SLE), especially those related to fundoscopy.

Method: A descriptive study of 41 lupus patients selected at random from May to July 1999 at the Collagen Disease Outpatient Clinic of the Rheumatology Service of the University Hospital, Federal University of Paraná. A protocol of clinical and ophthalmologic evaluation was applied.

Results: Forty-one patients were included in the study; 40 were females $(97.56 \%)$ and one was a male $(2.44 \%)$. Mean age was 32.22 years (range: 16 to 54 years). Thirty-seven patients $(87.80 \%)$ were white, two $(4.89 \%)$ were black, and three $(7.32 \%)$ were mulattoes. Time since SLE diagnosis ranged from two months to 18 years. Nineteen of the 41 patients $(46.34 \%)$ presented fundoscopic alterations related to SLE. The major lesions detected were cotton-wool spots and arteriolar narrowing (68.42\%) followed by increased excavation (10.52\%) and optic disk pallor (10.52\%), perivascular damage $(5.26 \%)$, and changes in the pigment epithelium of the retina $(5.26 \%)$.

Conclusion: There was a high prevalence of fundoscopic changes related to SLE, showing the importance of regular fundoscopic examinations even in asymptomatic lupus patients or in lupus patients with no associated diseases.

Keywords: Systemic lupus erythematosus; Fundoscopy.

\section{REFERÊNCIAS BIBLIOGRÁFICAS:}

1. Jabs DA, Miller NR, Newmann SA, Johnson MA, Stevens MB. Optic Neuropathy in Systemic Lupus Erythematosus. Arch Ophthalmol 1986;104:564-8.

2. Graham EM, Spalton DJ, Barnard RO, Garner A, Russell RW. Cerebral and Retinal Vascular Changes in Systemic Lupus Erythematosus. Ophthalmol 1985;92(3):444-8.

3. Yap EY, AnEong KG, Fong KY, Howe HS, Boey ML, Cheah WM, et al. Ophthalmic Manifestations in Asian Patientes with Systemic Lupus Erythematosus. Singapore Med. J. 1998;39(12):557-9.

4. Vine AK, Barr CC. Proliferative Lupus Retinopathy. Arch Ophthalmol 1984; 102:852-6.

5. Jabs DA, Fine SL, Hochberg MC, Newman SA, Heiner GG, Stevens MB Severe Retinal Vaso-Oclusive Disease in Systemic Lupus Erythematosus. Arch Ophthalmol 1986;104:558-63.

6. Pavan-Langston D. Manual de Oftalmologia: Diagnóstico e Tratamento. Rio de Janeiro: Medci; 1988.

7. Coppeto J, Lessell S. Retinopathy in Systemic Lupus Erythematosus. Arch Ophthalmol 1977;95:794-7.

8. Wong BAK, Everett AI, Jones JV. Visual loss as the initial symptom of systemic lupus erythematous. Am J Ophthalmol 1981;92:238-44.

9. Asherson RA, Merry P, Acheson JF, Harris EN, Hughes GR. Antiphospholipid antibodies: a risk factor for occlusive vascular disease in systemic lupus erythematous. Ann Rheumatic Diseases 1989;48:358-61.

10. Gold DH, Morris DA, Henkind P. Ocular findings in systemic lupus erythematous. Brit J Ophthal. 1972;56:800-4.

11. Mendes LE, Gonçalves JOR, Costa VP, Belfort Jr R. Alterações oculares no lupus eritematoso sistêmico. Arq Bras Oftalm 1998;61(6):713-6.

\title{
III CURSO ITINERANTE DE ESTRABISMO Promoção: Centro Brasileiro de Estrabismo
}

\section{DE DEZEMBRO DE 2000}

\author{
HOTEL MARIA DO MAR \\ FLORIANÓPOLIS • SC
}

Informações: Tels: (31) 3274-3555

c/ Dra. Maria de Lourdes M. M. Villas Boas 